\title{
TeV Gamma Rays from Ultrahigh Energy Cosmic Ray Interactions in the Cores of Active Galactic Nuclei: Lessons from Centaurus A
}

\author{
M. Kachelrie $\beta^{\mathrm{A}}$, S. Ostapchenko ${ }^{\mathrm{A}, \mathrm{B}}$, and R. Tomàs ${ }^{\mathrm{C}}$ \\ A Institutt for fysikk, NTNU, Trondheim, Norway \\ B D. V. Skobeltsyn Institute of Nuclear Physics, Moscow State University, Russia \\ ${ }^{\text {C }}$ II. Institut für Theoretische Physik, Universität Hamburg, Germany
}

Received 2009 October 11, accepted 2010 April 19

\begin{abstract}
TeV gamma rays have been observed from blazars as well as from radio galaxies like M 87 and Centaurus A. In leptonic models, gamma rays above the pair production threshold can escape from the ultrarelativistic jet, because large Lorentz factors reduce the background photon densities compared to those required for isotropic emission. Here we discuss an alternative scenario, where very high energy photons are generated as secondaries from ultrahigh energy cosmic rays interactions in the cores of active galactic nuclei. We show that $\mathrm{TeV}$ gamma-rays can escape from the core despite large infrared and ultraviolet backgrounds. For the special case of Centaurus A, we study whether the various existing observations from the far infrared to the ultrahigh energy range can be reconciled within this picture.
\end{abstract}

Keywords: gamma rays: theory — galaxies: individual (NGC 5128) — galaxies: active

\section{Introduction}

Centaurus A (Cen A or NGC 5128) is the nearest active galaxy, with a distance of only 3.8 Mpc (Rejkuba 2004). Photon emission from the nucleus of the galaxy has been detected in the radio, infrared (IR), X-ray, and in the $\mathrm{GeV}-\mathrm{TeV}$ range. Additionally, Abraham et al. (2007) reported two ultrahigh energy cosmic rays (UHECR) within $3.1 \mathrm{deg}$ around Cen $\mathrm{A}$ in the data from the Pierre Auger Observatory (PAO), thus offering the possibility for multi-messenger studies of this object. Such an effort has been undertaken e.g. by the present authors (Kachelrieß, Ostapchenko \& Tomàs 2009a). Assuming that Cen A accelerates protons to ultrahigh energy (UHE) and normalizing the obtained UHECR flux to the PAO observations, we predicted the accompanying gamma-ray and neutrino fluxes for two models: shock acceleration in the radio jet and acceleration in regular electromagnetic fields or shocks close to the core. Recent measurements of the $\mathrm{GeV}$ and $\mathrm{TeV}$ photon fluxes from Cen A by the Fermi-LAT (Cheung et al. 2009) and HESS (Aharonian et al. 2009) collaborations allowed us to exclude the former - photons are produced as secondaries in protonproton (pp) interactions in the radio jet, they can leave the source freely, and subsequent interactions with the extragalactic background light (EBL) result in a very flat $\mathrm{TeV}$ gamma-ray spectrum that is inconsistent with HESS data (Kachelrieß, Ostapchenko \& Tomàs 2009b). Moreover, the HESS data are consistent with a point source at the center of Cen A, although the relatively large angular resolution does not allow one to distinguish between the core and the inner jet as the $\mathrm{TeV}$ source. Note that the low flux of TeV gamma-rays observed from
Cen A does not allow to narrow down the source region studying the time-variability, as e.g. in the case M 87, where the strong time-variability observed (Acciari et al. 2009) excludes the outer jet region as the source of $\mathrm{TeV}$ photons, challenging even the knot HST-1 as the source. Moreover, the large Lorentz factors implied by the simplest self-synchrotron Compton models are often not supported by radio data (Piner, Pant \& Edwards 2008). While hadronic models for the origin of $\mathrm{TeV}$ gamma-rays have their own problems, they offer an alternative source of $\mathrm{TeV}$ photons that may partly avoid the problems of leptonic models.

There is a large variety of hadronic models proposed for Cen A or similar radio galaxies. There are models where $\mathrm{TeV}$ gamma-rays are produced in the immediate vicinity of the supermassive rotating Kerr black hole (Neronov \& Aharonian 2007; Rieger \& Aharonian 2008), in the inner jet via the proton-synchrotron model (Reimer, Protheroe \& Donea 2004; Orellana \& Romero 2009), or in the large-scale jet via proton-proton interactions (Hardcastle et al. 2008). Only the latter model is disfavoured by the HESS data.

We shall not consider hadronic models using the jet as an acceleration site (e.g. Reimer et al. 2004; Hardcastle et al. 2008; Orellana \& Romero 2009), but shall concentrate here on the second model discussed by Kachelrieß et al. (2009a). This model is based on hadronic acceleration near the core of the active galactic nucleus (AGN), and has the virtue of predicting $\mathrm{TeV}$ gamma-ray fluxes that are in good agreement with the later HESS observations. Moreover, this kind of model is in a sense complementary to leptonic models for acceleration 
close to the core: while in the latter models $\mathrm{TeV}$ photons can escape only for a low photon background in the central AGN region (Rieger \& Aharonian 2008, 2009), hadronic models require large ultraviolet (UV) and/or IR backgrounds for the efficient production of UHECR secondaries.

The aim of this article is twofold. First, we want to illustrate why TeV gamma-rays can escape in hadronic models from an AGN core, although the optical depth $\tau_{\gamma \gamma}$ is extremely large, $\tau_{\gamma \gamma}(E \sim \mathrm{TeV}) \sim 10^{3}$. To be more general,we consider various modifications of our original model, examining e.g. the influence of an additional IR background and a variation of the maximal energy $E_{\max }$ of the accelerated protons. Second, we apply our results to the special case of Cen A. In particular, we discuss if and how the various observations of Cen A from the far IR to the UHE range can be reconciled.

The outline of the paper is as follows. In Section 2, we recall our model in its original version that is characterized by the dominance of UV photons in $p \gamma$ and $\gamma \gamma$ interactions. In Section 3, we extend these calculations, varying the photon background density in the core region as well as $E_{\max }$. We summarize in Section 4.

\section{Ultraviolet Dominated Background}

We start by recalling the general features of our model; for more details refer to Kachelrieß et al. (2009a). Acceleration near the core of the AGN can proceed via shock acceleration in accretion shocks or via acceleration in regular fields. We do not describe the acceleration process microscopically but rather assume that acceleration to UHE takes place in the AGN core and produces a power-law flux of UHECRs, $\mathrm{d} N / \mathrm{d} E \propto E^{-\alpha}$. We performed calculations for different choices of $\alpha$ and for a broken power-law spectrum, with $\alpha_{1}=2.6$ for $E>10^{18} \mathrm{eV}$ and $\alpha_{2}=2$ for $E<10^{18} \mathrm{eV}$. In order to account for the PAO observations we set $E_{\max }=10^{20} \mathrm{eV}$ in this section.

The spectral energy distribution (SED) of AGNs partly shows a 'big blue bump' that is thought to be thermal emission in the UV range from an accretion disk. Because of the obscuring dust lane, the UV emission of Cen A cannot be observed directly, therefore we used the emission from an optically thick, geometrically thin accretion disk as a theoretical model (Shakura \& Syunyaev 1973). More specifically, we used $M=1 \times$ $10^{8} M_{\odot}$ for the mass of the black hole, $\dot{M}=6 \times$ $10^{-4} M_{\odot} \mathrm{yr}^{-1}$ for the accretion rate (Evans et al. 2004) and chose the radius of the last stable orbit for a Schwarzschild black hole $R_{0}=3 R_{\mathrm{s}}=9 \times 10^{13} \mathrm{~cm}$ as the smallest radius of the acceleration and emission region. Since the surface brightness drops fast with the radius, most of the radiation is emitted close to the core. To simplify the treatment, we described the emission region as a sphere of radius $R_{1}=15 R_{\mathrm{s}}$ filled with a homogeneous, isotropic photon field of constant density $n_{\mathrm{UV}}(\varepsilon)$, as discussed in more detail in Kachelrieß et al. (2009a). In addition, we assume that a hot corona produces an X-ray component with $n_{\mathrm{X}}(\varepsilon) \sim \varepsilon^{-1.7}$. The photon densities $n_{i}(\varepsilon)$ are normalized from the relation:

$$
L_{i}=\pi R_{1}^{2} c \int d \varepsilon \varepsilon n_{i}(\varepsilon)=\eta_{i} \dot{M} c^{2},
$$

using the measured luminosity $L_{\mathrm{X}}=4.8 \cdot 10^{41} \mathrm{erg} \mathrm{s}^{-1}$ in the 2-10 keV range for X-ray photons (Evans 2004; Markowitz 2007) and choosing the efficiency $\eta_{\mathrm{UV}}=10 \%$ in the UV range. Finally, we assumed that IR photons are distributed on a much larger spatial scale such that the corresponding density $n_{\mathrm{IR}}(\varepsilon)$ can be neglected.

Photons, electrons, and positrons (electromagnetic [EM] particles) produced in $p \gamma$ interactions further cascade on the UV photon background via pair production, inverse Compton scattering, and synchrotron emission. The latter contribution to the cascade, due to the regular and turbulent magnetic fields, requires a separate discussion - electrons move along the regular field lines, emitting synchrotron and curvature radiation. We describe these processes by an effective random magnetic field. Because of the rather high energies of the electrons involved, synchrotron emission proceeds in the quantum regime, i.e. with $\chi \equiv e B_{\perp} E_{e} / m_{e}^{3} \gtrsim 1$. Consequently, electrons emit a small number of high-energy photons instead of producing a semi-continuous spectrum of low energy photons. Thus, the cascade develops in a very peculiar way: photons are pair-producing on the background photons, while electrons contribute mainly to the cascade via synchrotron radiation. We verified that the resulting spectrum depends only weakly on the assumed strength of the effective magnetic field strength $B$. Changing $B$ by four orders of magnitude from $10^{-2}$ up to $10^{2} \mathrm{G}$ has only a minor impact on final photon spectra leaving the source. ${ }^{1}$

The predicted photon spectra after cascading in the EBL are compared to recent HESS and preliminary Fermi-LAT data for three different choices of injection proton spectra in Figure 1. Remarkably, the obtained spectral shape in the $\mathrm{TeV}$ range depends only weakly on the proton spectral slope and agrees well with the HESS data for all the three cases considered. However, the broken power-law proton spectrum results in a too high photon flux in the $\mathrm{GeV}$ range, in contradiction to the Fermi data.

Let us now discuss the feature of our numerical results that may be most interesting from a general point of view. How can TeV gamma-rays leave the AGN core despite the large UV density? To illustrate this problem, in Figure 2 we plot as a red line the optical depth $\tau_{\gamma \gamma}(E)$ as a function of energy for a photon propagating through the distance range $\left[R_{0}, R_{1}\right]$ corresponding to the UV emission 'photosphere'. The optical depth is of order $10^{3}$ in the TeV

\footnotetext{
${ }^{1}$ For high-energy electrons, the characteristic parameter for the synchrotron emission $\chi$ remains large over a wide range of magnetic field strengths, which thus assures that the emission proceeds in the quantum regime.
} 


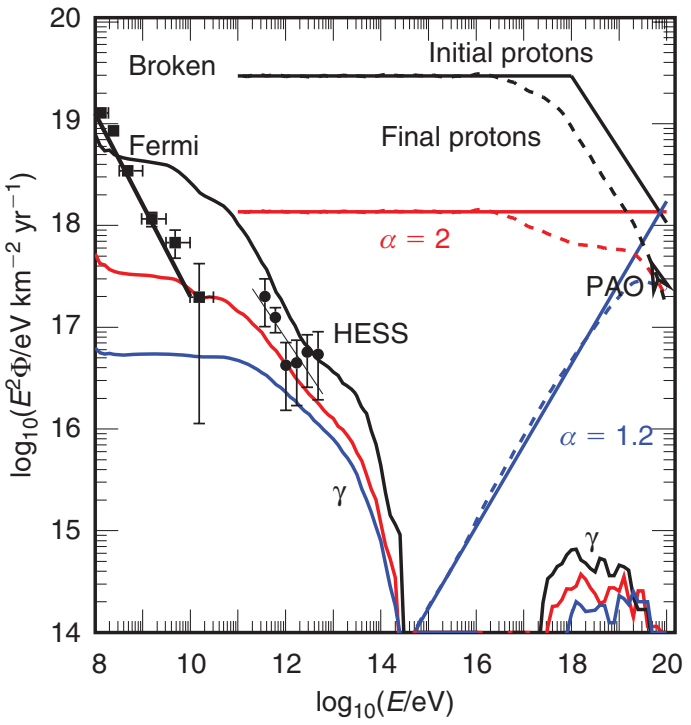

Figure 1 Photon (solid lines) and proton (dashed) fluxes from Cen A, normalized to the PAO results, for different proton injection spectra (solid): broken power-law (black), power-law with $\alpha=2$ (red), and power-law with $\alpha=1.2$ (blue), compared to the data of HESS and Fermi-LAT.

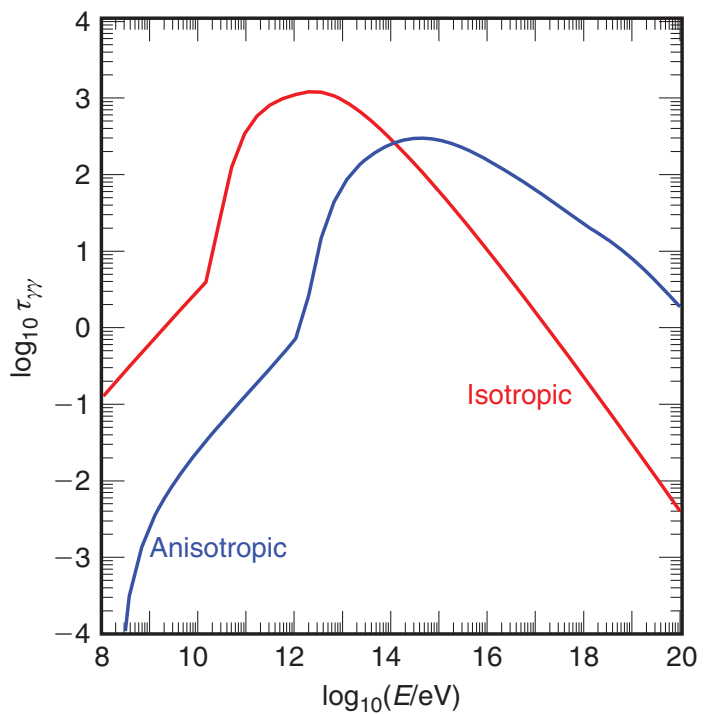

Figure 2 The optical depth $\tau_{\gamma \gamma}$ for photon-photon interactions inside the $\mathrm{UV}$ emission region $\left(r<R_{1}\right.$, red line) and outside in the quasi-collinear regime ( $r>4 R_{1}$, blue line) only on UV photons.

range and one expects that most electromagnetic energy cascades down in the $10 \mathrm{GeV}$ range, forming a characteristic spectral plateau.

There are two different contributions to the final $\mathrm{TeV}$ photon flux. To understand the first one, it is useful to consider separately the development of the EM cascade inside $\left(R<R_{1}\right)$ and outside $\left(R>R_{1}\right)$ the emission region. In the former case, the angular distribution of UV photons is isotropic. The emission photosphere is opaque for $\mathrm{TeV}$ gamma-rays. However, UHE photons $\left(E \gtrsim 10^{18} \mathrm{eV}\right)$ interact with the background photon fields in the deep Klein-Nishina regime $\left(E \varepsilon \gg s_{\mathrm{thr}}=4 m_{e}^{2}\right)$. Since the pair production cross section decreases as $1 / E$ above the threshold, the core region appears to be transparent for them. Outside the emission region UV photons are anisotropic. At large distances $r \gg R_{1}$ their density goes down as $n_{\mathrm{UV}} \sim\left(R_{1} / r\right)^{2}$ and their momentum vectors obey

$$
\cos \theta=\mathbf{p} \cdot \mathbf{r}|\mathbf{p}||\mathbf{r}| \geq 1-\left(R_{1} / r\right)^{2} .
$$

Thus $\mathbf{p}$ becomes more and more collinear with the momentum vector of the outgoing EM cascade particles. At large enough distances, UHE photons interact therefore with the UV background close to the threshold, $s$ $=2 E \varepsilon(1-\cos \theta) \sim 4 m_{e}^{2}$, and the produced $\mathrm{TeV}$ photons leave the source vicinity freely. To illustrate this picture, we also show in Figure 2 the optical depth for a photon injected at $r=4 R_{1}$ as a blue line.

The second contribution to the final $\mathrm{TeV}$ photon flux are muons created in $p \gamma \rightarrow n \pi^{+}$reactions. Their decay length for $E \approx 10^{18} \mathrm{eV}$ becomes comparable to the size $R_{1}$ of the isotropic UV region. Thus most UHE muons decay outside in the 'collinear region', where the cascade stops above the $\mathrm{TeV}$ scale.

In summary, the $\mathrm{TeV}$ gamma-ray flux is formed by decays of UHE muons and by re-shuffling UHE photons with $E \gtrsim 10^{17} \mathrm{eV}$ in the anisotropic UV background surrounding the source region.

It is worth stressing the salient points of this picture. First, both processes require a sufficiently high flux of UHE photons or muons. Such particles can only be generated as secondaries from accelerated hadrons. Second, the observed gamma-ray spectrum is essentially formed in the source vicinity. Subsequent interactions with the EBL lead only to minor modifications. Third, the EM cascade in the source vicinity, which re-shuffles photons of energies $E \gtrsim 10^{18} \mathrm{eV}$ to the $\mathrm{TeV}$ range, includes a large number of particle generations (emission steps). As a consequence, the photon spectral shape in the $\mathrm{TeV}$ range depends rather weakly on the proton injection spectrum; the latter rather defines the normalization of the spectral 'plateau' in the $\mathrm{GeV}$ range.

By contrast, photons leave the source freely when they are produced by protons accelerated over long distances $(\sim \mathrm{kpc})$ in the radio jet. The further re-shuffling of UHE photons in interactions with the EBL results in a very flat $\mathrm{TeV}$ gamma-ray spectrum (Kachelrieß et al. 2009a), in contradiction to the HESS observations.

\section{Varying the Photon Background and $\boldsymbol{E}_{\max }$}

Several of the assumptions underlying our analysis in the last section were chosen on the basis of weak empirical evidence. In detail, these assumptions are:

1. A negligible impact of the IR photons on the cascade development.

2. The large value of $E_{\max }=10^{20} \mathrm{eV}$.

3. The size of the UV bump, with an efficiency of $\eta_{\mathrm{UV}}=10 \%$, as defined in Equation (1). 


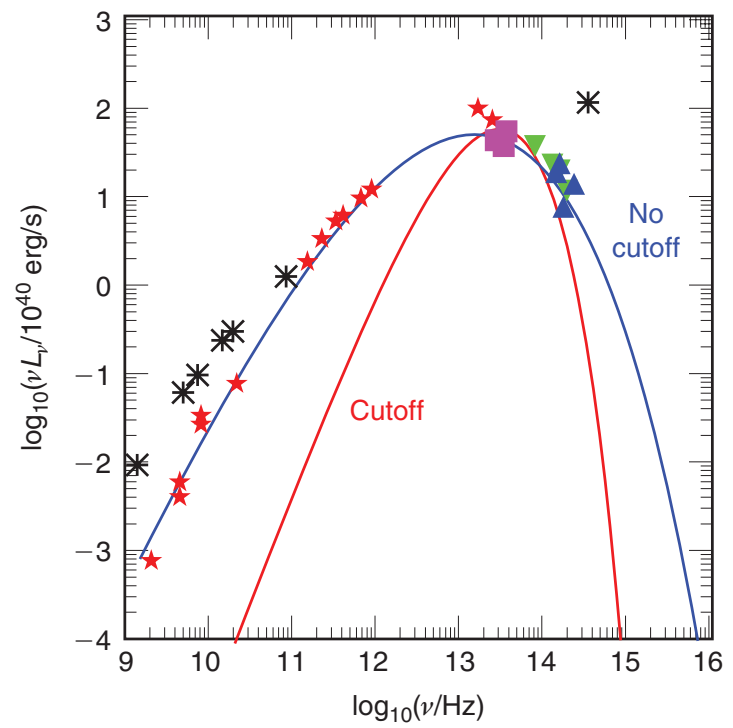

Figure 3 Parametrizations of the IR part of the SED towards smaller frequencies, (i) cutoff (red), and (ii) no cutoff (blue) in the far IR, together with observational data points.

The objective of this section is to discuss how the results obtained in Kachelrieß et al. (2009a), and summarized in Figure 1, are modified by relaxing the previous assumptions, i.e., varying the background photon field of the source as well as $E_{\max }$. In the following, we shall use the present estimate $M=5 \times 10^{7} M_{\odot}$ for the black hole mass of Centaurus A (Cappellari et al. 2008), which corresponds to the inner radius of the acceleration/ emission region $R_{0} \simeq 4.5 \times 10^{13} \mathrm{~cm}$. We shall always use a $\mathrm{d} N / \mathrm{d} E \sim E^{-2}$ spectrum for the injected protons, and if not otherwise stated $\eta_{\mathrm{UV}}=5 \%$.

\subsection{Infrared Background}

We start by investigating the effect of varying the IR background on the $\mathrm{TeV}$ photon spectra. Conventionally, the dominant contribution to the SED in the IR range is supposed to be formed by the thermal emission from the dust torus and is thus distributed on multi-parsec scales. Nevertheless, recent interferometry measurements by Meisenheimer et al. (2007) indicate that a significant part of the near IR emission (from $60 \%$ at $\lambda=13 \mu \mathrm{m}$ up to $80 \%$ at $\lambda=8 \mu \mathrm{m}$ ) is due to a compact unresolved source of size $R_{\mathrm{IR}}<0.2 \mathrm{pc}$. (Note, however, that those results are challenged by Radomski et al. 2008.) Moreover, it has been speculated by Meisenheimer et al. (2007) that the whole SED from the IR down to the radio range is mainly generated by a tiny synchrotron source of the size $R_{\mathrm{IR}} \sim 0.01 \mathrm{pc}$. Since the optical depth scales as $\tau \propto R^{-1}$, interactions with IR photons close to the core should seriously modify the picture discussed in Section 2.

In order to analyze the phenomenological consequences of an IR background on the TeV photon spectra, we consider two different parametrizations of the SED shown in Figure 3: (i) with a cutoff in the far IR, as one

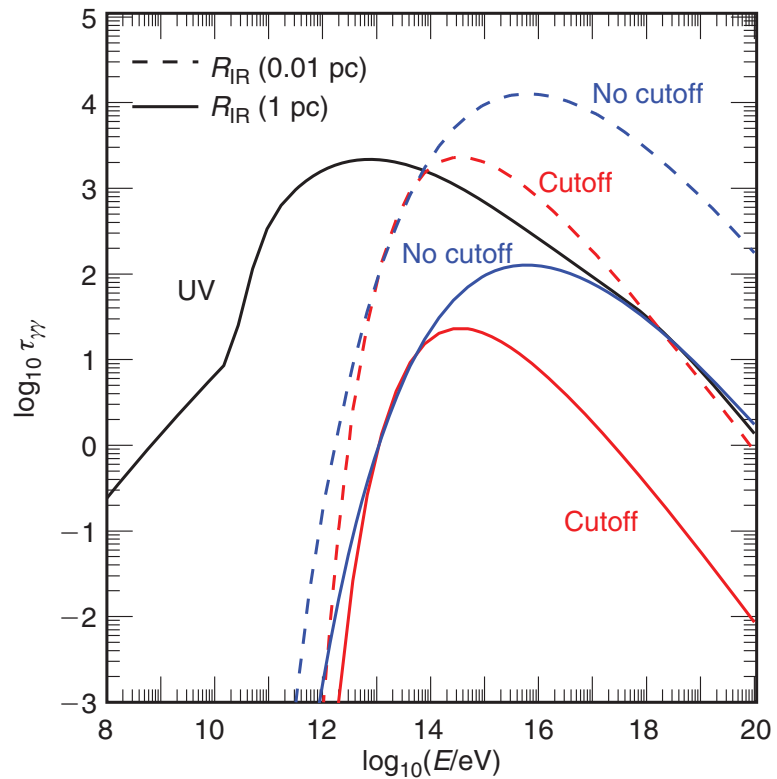

Figure 4 Photon optical depth due to interactions with the UV (solid black line) and IR backgrounds for different parametrizations of the infrared part of the SED: cutoff (blue), and no cutoff (red); for $R_{\mathrm{IR}}=0.01$ (dashed) and $1 \mathrm{pc}$ (solid).

expects if IR photons are due to thermal emission of the torus (Marconi et al. 2000); (ii) the whole SED from the IR down to the radio range emitted by the same source of size $R_{\mathrm{IR}}$ as suggested by Meisenheimer et al. (2007). Additionally, we have varied the spatial size of the IR source over two orders of magnitude, $R_{\mathrm{IR}}=1,0.1$ and $0.01 \mathrm{pc}$.

Before showing the results, let us first discuss how the presence of the IR background modifies the interaction depth of photons and protons. The relative size of the contribution of the (isotropic and anisotropic) UV and the IR backgrounds to the total optical depth of photons is illustrated for the two parametrizations of the SED and two source sizes, $R_{\mathrm{IR}}=1$ and $0.01 \mathrm{pc}$ in Figure 4 . Let us first consider the contribution to the optical depth due to photon interactions with the IR background in the UV emission region, i.e., at $R<R_{1} \approx 10^{-4} \mathrm{pc}$, which can be obtained by rescaling the different curves of Figure 4: $\tau_{\gamma \gamma}^{\mathrm{IR}}\left(R_{1}\right)=\tau_{\gamma \gamma}^{\mathrm{IR}}\left(R_{\mathrm{IR}}\right) R_{1} / R_{\mathrm{IR}}$. For most of the cases considered, photon interactions with IR photons at $R<R_{1}$ are a small correction compared to interactions with the UV background. Thus, the situation is analogous to the one discussed previously: photons created in the UV region with energies above $10^{18} \mathrm{eV}$ can escape. Once they enter the IR region they cascade down to the $10 \mathrm{TeV}$ energy range, now both due to interactions with quasi-collinear UV photons and with the IR photons; see Figure 2. Therefore the reshuffling mechanism still works. This is true except for the case of an extreme compact IR background that explains the full SED, i.e. $R_{\mathrm{IR}}=0.01 \mathrm{pc}$ without cutoff. In this case, interactions with IR photons dominate even inside $R_{1}$ and make the UV region almost opaque for photons with energies below $E \approx 10^{20} \mathrm{eV}$. 
As far as the proton interactions with the UV and IR background are concerned, we show in Figure 5 the interaction lengths for $p \gamma$ pion interactions assuming $R_{\mathrm{IR}}=0.01 \mathrm{pc}$ compared to the size of the UV region $R_{1}$ and the muon decay length. The threshold energy for $p \gamma_{\mathrm{UV}}$ and $p \gamma_{\mathrm{IR}}$ is roughly $10^{16} \mathrm{eV}$ and $10^{18} \mathrm{eV}$, respectively. For this choice of parameters, the interaction depth $\tau_{p \gamma}$ above the threshold is of order a few for both photon backgrounds, while rescaling e.g. to $R_{\mathrm{IR}}=1 \mathrm{pc}$ gives $\tau_{p \gamma} \propto R^{-1} \sim$ few $\%$ in the IR region. Figure 5 also shows

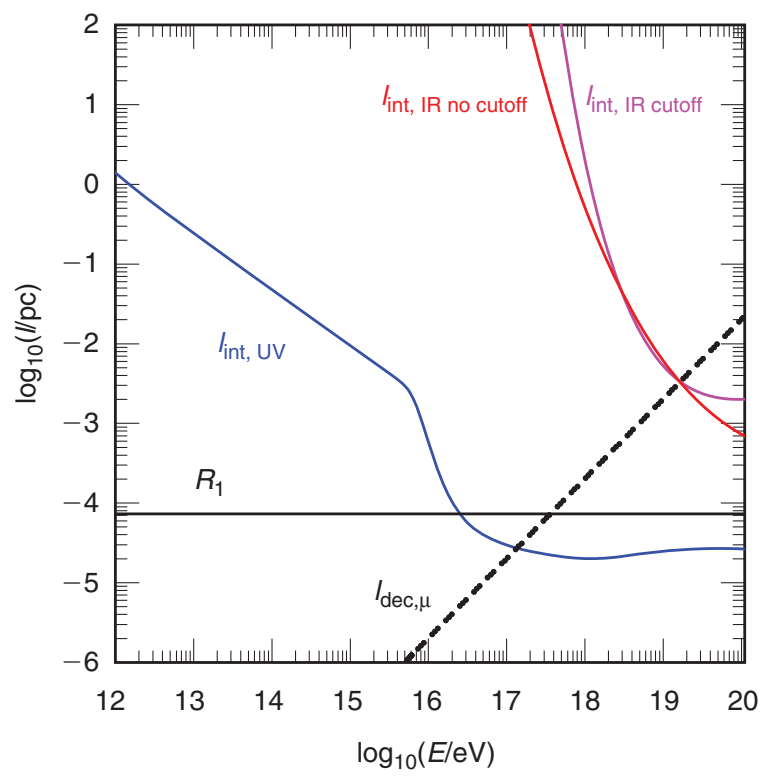

Figure 5 Comparison of the size of the UV source $\mathrm{R}_{1}$ (black solid line) with the interaction length for $p \gamma$ scattering on UV (blue solid) and IR photons with cutoff (solid magenta) and no cutoff (solid red), together with the muon decay length (black dashed line), for $\eta_{\mathrm{UV}}=5 \%$ and $R_{\mathrm{IR}}=0.01 \mathrm{pc}$.

(a)

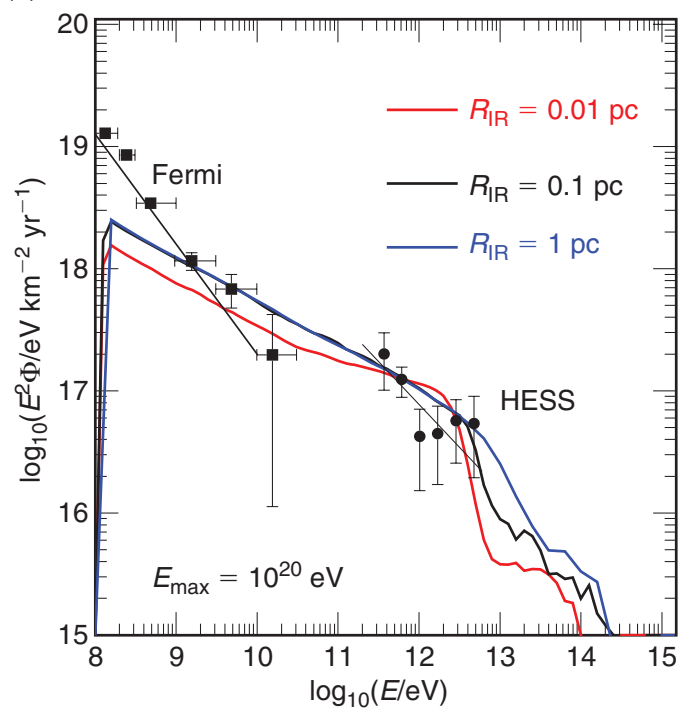

that muons with energies above a few $\times 10^{17} \mathrm{eV}$ escape from the UV region before decaying.

We now discuss our new results for the photon spectrum from Cen $\mathrm{A}$ and, in particular, if our numerical results agree with our expectations from the $\tau_{\gamma \gamma}$ and $l_{p \gamma}^{\text {int }}$ plots. In all cases we normalize the spectra to the HESS results. In Figure 6, photon fluxes calculated for the maximal UHECR energy $E_{\max }=10^{20} \mathrm{eV}$ are plotted for three different sizes of the IR source, with a cutoff in Figure $6 a$ and no cutoff in Figure 6b. Compared to case of only UV background shown in Figure 1, the cutoff observed in the gamma ray flux is more pronounced and shifted to lower energies. Still the shape is consistent with the HESS data, except perhaps for the most drastic case, $R_{\mathrm{IR}}=0.01 \mathrm{pc}$ without a cutoff. For all the cases considered, the obtained spectra do not contradict the Fermi data in the GeV range.

How could we obtain a substantial $\mathrm{TeV}$ photon flux, although the core region is completely opaque for UHE photons? There are again two effects that explain the TeV photon flux. First, the probability for $p \gamma$ interactions on IR photons is non-negligible, and for some parameters even comparable to the one on UV, see Figure 5. Since $\tau_{p \gamma} \sim \max \left\{\tau_{\gamma \gamma}\right\} / 2000$, the distribution of the starting points for these cascades is shifted outwards and the remaining path length through the photon gas thus reduced. Second, the muon mechanism already encountered in the 'UV only' case is again operative. The relative importance of these two effects depends on the chosen parameters, in particular the size of the IR and UV regions, the shape of the IR cutoff as well as $E_{\max }$.

In order to understand this better, we show in Figure $6 \mathrm{~b}$ for the case $R_{\mathrm{IR}}=0.01 \mathrm{pc}$ not only the total photon flux, but separately by a dashed red line those photons that were generated by electromagnetic cascades initiated outside the UV emission region, i.e., at $R>R_{1}$. Note that this includes EM cascades initiated by decaying muons as

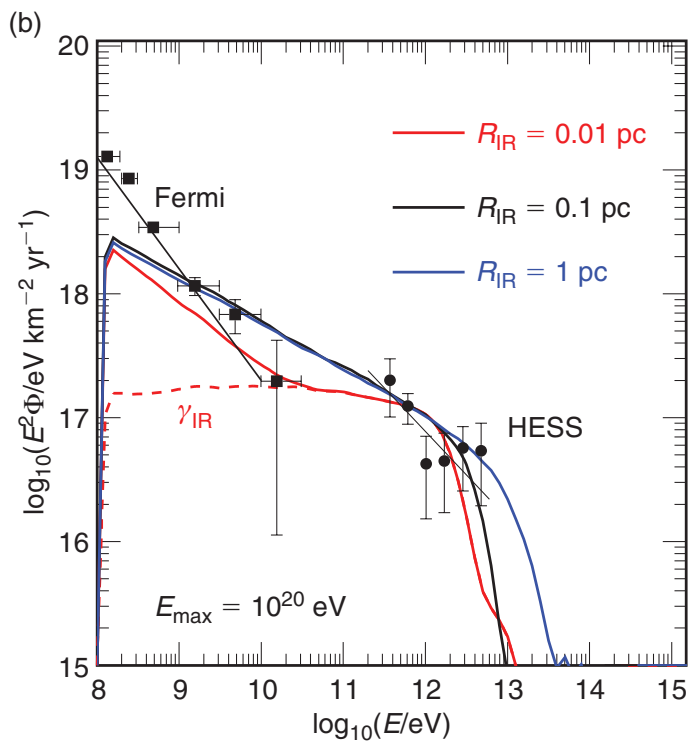

Figure 6 Photon fluxes from Cen A normalized to the HESS observations for different sizes of the IR source and for different parametrizations of the SED: (a) cutoff in the far IR, (b) no cutoff in the far IR (right); $E_{\max }=10^{20} \mathrm{eV}$. 
well as protons interacting at $R>R_{1}$. Clearly, the flux above $1 \mathrm{TeV}$ is dominated by photons that are created as final products of EM cascades initiated outside the UV photosphere.

It is remarkable how similar the results presented in Figure 6 are, being practically independent on the spectral shape and the spatial extension of the IR background. The amount of $\mathrm{TeV}$ photons produced depends on the flux of photons generated in the UV region at energies above $10^{18} \mathrm{eV}$ and being reshuffled in the source vicinity (at $R>R_{1}$ ), of photons originating from proton-gamma interactions at $R>R_{1}$, which come mainly from the decays of produced neutral pions, and of high energy muons created in the UV emission region and decaying outside it. Whereas the latter contribution is only determined by $\eta_{\mathrm{UV}}$ and $R_{1}$, the two former ones explicitly depend on the properties of the IR background. However, EM cascades stop around $10 \mathrm{TeV}$ for all the IR backgrounds considered, see Figure 4. This value corresponds roughly to the cutoff visible in Figure 6.

\subsection{Maximum Energy}

Deriving the previous results, a maximum energy of $10^{20} \mathrm{eV}$ for the protons was assumed. Acceleration of protons to these energies requires magnetic field strengths of order $B \sim \mathrm{kG}$. This is an order of magnitude higher than what one would expect from equipartition arguments, $B^{2} /(8 \pi) \sim L /\left(4 \pi R_{1}^{2} c\right)$, and two orders above the estimate developed by Bicknell \& Li (2007) using a Poynting flux dominated jet. An enhancement of the field strength may, in principle, be expected due to the back-reaction of accelerated (Bell \& Lucek 2001). However, alternative pictures - where the core region acts as a pre-accelerator, producing protons up to $E_{\max } \sim 10^{18} \mathrm{eV}$, while acceleration to UHE takes place in the lobes (Hardcastle et al.

(a)

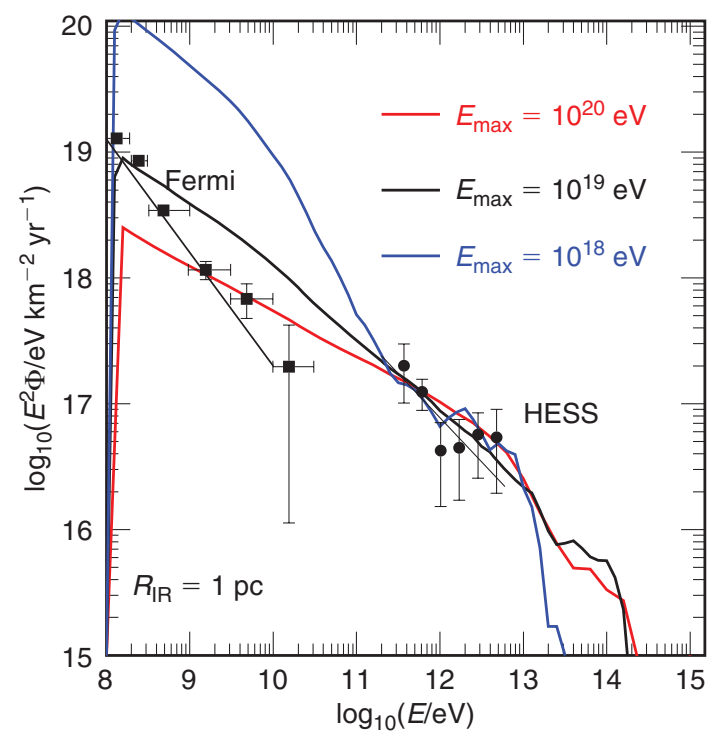

2008) or Cen A produces no UHECRs at all - are certainly also possible.

In this subsection we analyze the consequences of lowering the maximal energy. In Figure 7, we show the photon fluxes for three different values of the maximal UHECR energy, $E_{\max }=10^{18}, 10^{19}$ and $10^{20} \mathrm{eV}$, and for two different scenarios for the IR background. Reducing $E_{\max }$ leads to a smaller number of UHECR above the threshold for interactions on the IR and a smaller number of muons decaying outside, reducing in turn the $\mathrm{TeV}$ photon flux. Thus keeping the $\mathrm{TeV}$ flux fixed, the $\mathrm{GeV}$ flux increases, exceeding the Fermi data for $E_{\max }=$ $10^{18} \mathrm{eV}$ and marginally for $E_{\max }=10^{19} \mathrm{eV}$.

\subsection{Ultraviolet Background}

The presence of a blue/UV bump in the SED spectrum is a general characteristic of AGNs. In the analysis reported in Kachelrieß et al. (2009a), the luminosity of the UV bump was set to $10 \%$ of the accretion rate. Because no observational data for Cen A exist in this range, the UV emission could be considerably smaller than we assumed. This is also suggested by the low accretion rate of Cen A, that indicates that the accretion is advection-dominated.

Therefore, we analyze in the following the consequences of modifying the UV background. Reducing the relative importance of the UV background should lead to a larger fraction of $p \gamma$ interactions on the IR, thereby enhancing the $\mathrm{TeV}$ and reducing the GeV flux. This effect is demonstrated in Figure 8, where the photon fluxes for $\eta_{\mathrm{UV}}=0.1 \%$ are shown for two IR backgrounds and $R_{\mathrm{IR}}=1 \mathrm{pc}$. The GeV photon flux is now, as expected, suppressed and even for $E_{\max }=10^{18} \mathrm{eV}$ more or less consistent with Fermi data, apart from a flatter than observed spectral shape. This shape coincides with the flat contribution from the $R>R_{1}$ expected in the case

(b)

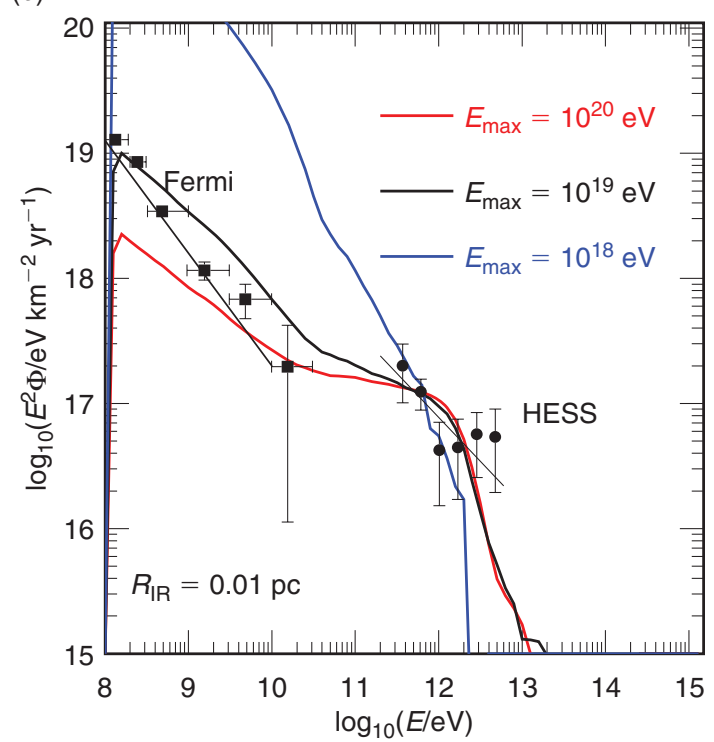

Figure 7 Photon fluxes from Cen A calculated for different $E_{\max }$ and (a) for a cutoff in the far IR and $R_{\mathrm{IR}}=1 \mathrm{pc}$, (b) without cutoff and $R_{\mathrm{IR}}=0.01 \mathrm{pc}$. 
(a)

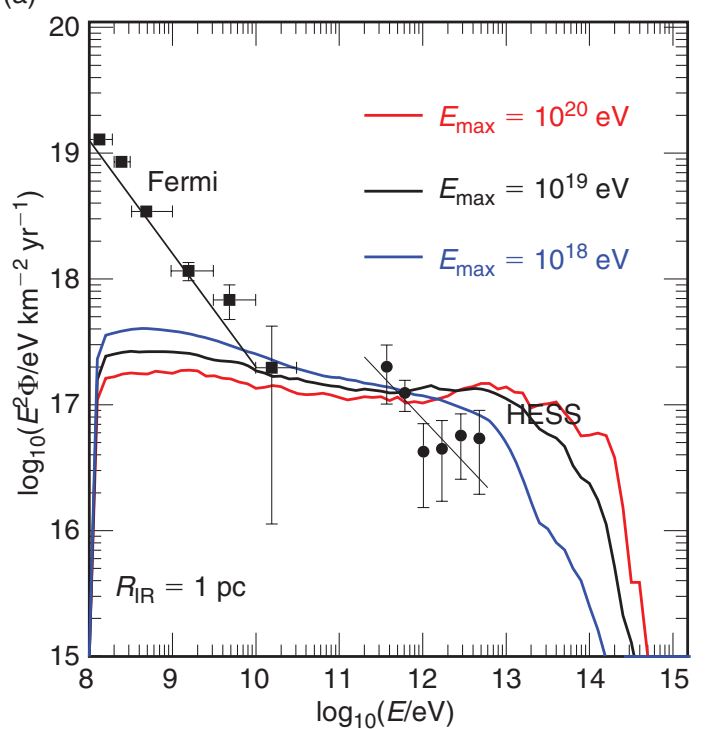

(b)

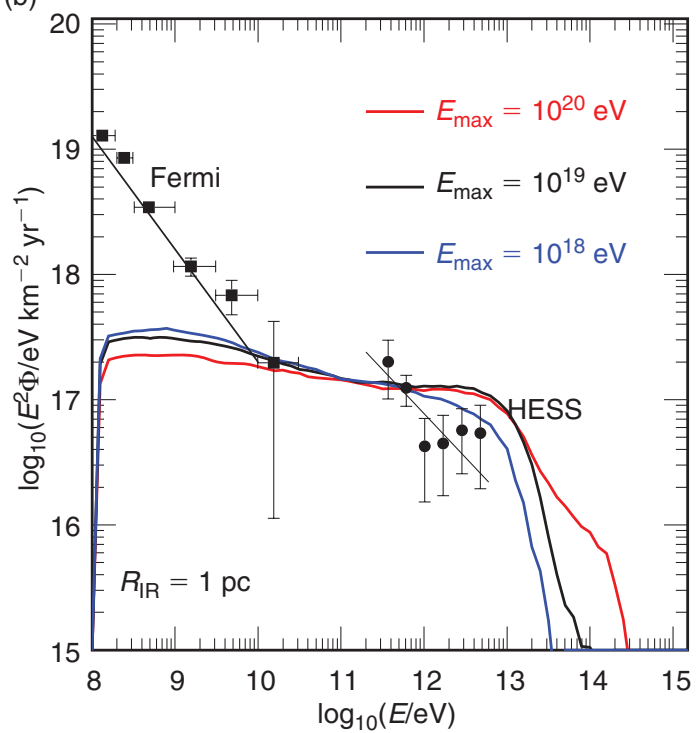

Figure 8 Photon fluxes from Cen A for $R_{\mathrm{IR}}=1 \mathrm{pc}$ and $\eta_{\mathrm{UV}}=0.1 \%$ : (a) cutoff in the far IR; and (b) without the cutoff in the far IR.

with important UV component; see dashed lines in Figure $6 \mathrm{~b}$. Both with and without cutoffs, one would expect from the criterion $\tau_{\gamma \gamma}=1$ that no photons with $E \gtrsim$ few $\mathrm{TeV}$ should be observed from Cen A, while the actual flux for these parameters extends up to few hundreds of $\mathrm{TeV}$. The extension to high energies is most prominent for a cutoff in the IR and large $E_{\max }$. Both conditions lead to a larger fraction of UHE protons with energy above the pion production threshold interacting with the IR photons, and thus to the generation of EM cascades at relatively large distances from the core.

However, there is a price to be paid for the suppression of the GeV photon flux: since $\tau_{p \gamma}$ is now only of order $5 \%$ above the threshold, a larger cosmic ray (CR) flux is required to fit the HESS data. In particular, the CR luminosity required for the parameters used in Figure 8a is $L_{\mathrm{CR} \text {,tot }}=2 \times 10^{43} \mathrm{erg} \mathrm{s}^{-1}$ using $E_{\min }=10^{9} \mathrm{eV}$. This value should be compared to the total bolometric luminosity of Cen A, $L_{\mathrm{bol}}=10^{44} \mathrm{erg} \mathrm{s}^{-1}$. Hence such a scenario requires an uncomfortably large fraction of the total energy output of the AGN core channeled into CRs.

This problem can be ameliorated reducing the size of the IR source. Choosing $R_{\mathrm{IR}}=0.01 \mathrm{pc}$, the interaction depth $\tau_{p \gamma}$ in the IR region increases to $\tau_{p \gamma} \sim$ few above the threshold and as a result the CR luminosity is reduced. In the particular case of an IR background without cutoff in the far IR, the required CR luminosity is a factor of five smaller, $L_{\mathrm{CR}, \text { tot }}=4 \times 10^{42} \mathrm{erg} \mathrm{s}^{-1}$ for $E_{\min }=10^{9} \mathrm{eV}$, but the cutoff is in the $10 \mathrm{TeV}$ range. Thus we predict an extension of the very high energy (VHE) photon flux from Cen $\mathrm{A}$ into the $100 \mathrm{TeV}$ range, only if the UV background in Cen $A$ is sufficiently large, $\eta_{\mathrm{UV}} \gtrsim 1 \%$.

\section{Summary}

We have investigated the hypothesis that the TeV photons observed by HESS from Cen A are produced as secondaries in hadronic interactions. Varying the parameters of the photon background in the IR and UV, we have identified cases for which the predicted VHE photon spectrum extends beyond the energy cutoff predicted naively from the condition $\tau_{\gamma \gamma}\left(E_{\text {cut }}\right)=1$. Such cases can be as diverse as (i) a dominant UV background with a sufficiently diffuse IR background or (ii) a low UV background with a compact IR background on a (sub-)pc scale. Applied to Cen A, both cases predict photon spectra that are compatible with HESS and Fermi data, although for the second option the obtained spectral shape in the $\mathrm{TeV}$ range is significantly flatter than observed experimentally. Additionally, the first case is also consistent with the possibility of Cen A as an Auger source. Within our scenario, the cutoff in the VHE photon flux from Cen $\mathrm{A}$ could be in the $10 \div 100 \mathrm{TeV}$ range, if (i) $E_{\max } \gtrsim 10^{19} \mathrm{eV}$ and (ii) either the UV background in Cen A is sufficiently large, $\eta_{\mathrm{UV}} \gtrsim 1 \%$, or the source of the IR background is extremely compact, $R_{\mathrm{IR}} \sim 0.01 \mathrm{pc}$.

Depending on the parameters, different mechanisms contribute to the photon flux in the multi-TeV region: UHE muons created $p \gamma \rightarrow \pi^{+} n$ reactions can carry EM energy efficiently outwards befor UHE photons interact in the inner part of the AGN core in the Klein-Nishina regime, therefore also efficiently channeling EM energy before they interact in the collinear regime. Finally, $p \gamma$ interactions on IR photons additionally enhance the highenergy tail of the photon flux. All three mechanisms are based on hadronic primaries and require a flux of UHECRs extending at least to $10^{18} \mathrm{eV}$.

Viable combinations of IR and UV backgrounds are those that allow a sufficiently large fraction of UHE photons or muons to traverse the UV region and/or that lead to an adequate number of $p \gamma$ interactions on the IR, while satisfying luminosity constraints.

Finally, we stress that our findings are not only valid for Cen A, but could be applied more generally to other 
AGN as sources of VHE energy photons and in particular to blazars.

\section{Acknowledgments}

We would like to thank M. J. Hardcastle for useful discussions. S.O. acknowledges a Marie Curie IEF fellowship from the European Community, R.T. partial support from the Deutsche Forschungsgemeinschaft within the SFB 676.

\section{References}

Abraham, J. et al., 2007, Science, 318, 939

Acciari, V. A. et al., 2009, Science, 325, 444

Aharonian, F. et al., 2009, ApJL, 695, L40

Aharonian, F. \& Rieger, F., 2009, A\&A, 506, 3, L41

Bell, A. R. \& Lucek, S. G., 2001, MNRAS, 321, 438

Berezinsky, V., Gazizov, A. Z. \& Grigorieva, S. I., 2004, Nucl. Phys. Proc. Suppl., 136

Bicknell, G. V. \& Li, J., 2007, Ap\&SS, 311, 275
Cappellari, M. et al., 2008, arXiv:0812.1000 [astro-ph]

Cheung, T. et al., 2009, PASA, this volume

Evans, D. A. et al., 2004, ApJ, 612, 786

Hardcastle, M. J., Cheung, C. C., Feain, I. J. \& Stawarz, L., 2008, MNRAS, 24, 337

Kachelrieß, M., Ostapchenko, S. \& Tomàs, R., 2009a, New J. Phys., 11,065017

Kachelrieß, M., Ostapchenko, S. \& Tomàs, R., 2009b, Int. J. Mod. Phys. D, 18, 1591

Marconi, A. et al., 2000, ApJ, 528, 276

Markowitz, A. et al., 2007, ApJ, 665, 209

Meisenheimer, K. et al., 2007, A\&A, 471, 453

Neronov, A. \& Aharonian, F. A., 2007, ApJ, 671, 85

Orellana, M. \& Romero, G. E., 2009, AIP Conf. Proc., 1123, 242

Piner, B. G., Pant, N. \& Edwards, P. G., arXiv:0801.2749 [astro-ph] Radomski, J. T. et al., 2008, ApJ, 681, 141R

Reimer, A., Protheroe, R. J. \& Donea, A. C., 2004, New Astron. Rev., 48, 411

Rejkuba, M., 2004, A\&A, 413, 903

Rieger, F. M. \& Aharonian, F. A., 2008, A\&A, 479, L5

Shakura, N. I. \& Syunyaev, R. A., 1973, A\&A, 24, 337 